ISSN: 0212-0267

DOI: http://dx.doi.org/IO.I420I/hedu2OI534287303

\title{
EN LA FRONTERA: SEÑAS DE IDENTIDAD \\ DE LA LABOR PEDAGÓGICA \\ HISPANO-AMERICANISTA \\ EN MARÍA DE MAEZTU (1924-1936)
}

\section{In the frontier: identity traits in Maria de Maeztu's pedagogical Hispanic-American activity (I924-I936)}

\author{
Elvira M. MELIÁN \\ Universidad Rey Juan Carlos \\ Correo-e: emelian@telefonica.net
}

Recepción: 24 de abril de 20I4. Envío a informantes: 30 de mayo de 2014.

Fecha de aceptación definitiva: I de agosto de 2014

Resumen: Las mujeres españolas somos deudoras de la pionera labor pedagógica en la que María de Maeztu consumió gran parte de su vida. Frente a los numerosos estudios sobre su obra en España, se olvida con frecuencia su papel como adalid de una corriente educativa hispano-americanista cristiana femenina que, durante el primer tercio del siglo xx, persiguió el progreso cultural de la mujer a ambos lados del Atlántico. El análisis de sus ensayos y conferencias sobre esta temática, y de la correspondencia con otras partícipes del mismo, nos permite recuperar la íntima naturaleza de un movimiento estrechamente ligado a la idiosincrasia de unas mujeres que apostaron, hasta su último aliento, por tan compleja como admirable empresa.

Palabras Clave: María de Maeztu; educación de la mujer; Residencia de Señoritas; Junta para Ampliación de Estudios e Investigaciones Científicas; Pedagogía; hispano-americanismo femenino cristiano.

AвSTRACT: Spanish women are due to pedagogical efforts that consumed a huge part of María de Maeztu's life. In opposition to the profuse studies about her Spanish work, there is a general unconsciousness about her leading role in the Christian-feminine educational strategy that, during the first third of the 2oth century, pursued the cultural flowering of Hispano-American women. By analyzing her 
essays and conferences about the subject, and by examining her correspondence with other remarkable participants, we recover the «inside nature» of a movement closely linked to its members fundamental beliefs.

Key words: María de Maeztu; women’s education; Residencia de Señoritas; Junta para Ampliación de Estudios e Investigaciones Científicas; Pedagogy; Christian feminine Hispano-Americanism.

\section{Introducción}

$\mathrm{E}$

N SEPTIEMbRe De I928 Carlos Morla LynCH, amigo y posterior biógrafo de Lorca, describe en su diario a la recién conocida María de Maeztu como una mujer rubia, de estatura menuda, carente de coquetería, alejada de la moda, nerviosa, vibrante y de gran locuacidad" «Una tarabilla, llena de criterio y de buen sentido", que declara en este primer encuentro su entusiasmo por la labor educadora «misión para la cual ha nacido», salvadas algunas neblinas provocadas por las ingratitudes humanas². Con 47 años, María se encuentra en el cénit de su proyección pública como representante del mundo de la enseñanza en la Asamblea Nacional Consultiva de Primo de Rivera y vocal de la Junta para Ampliación de Estudios e Investigaciones Científicas (JAE)³. Dirige además la Residencia de Señoritas y el grupo de niños de la Sección Preparatoria del InstitutoEscuela ${ }^{4}$. Con este bagaje defiende ante Morla Lynch y los demás contertulios,

una destrucción de todo lo establecido no con un espíritu de exterminio sino, por el contrario, inspirado en un sano anhelo de renovación completa sobre un plan más elevado y de mayor justicia para todos. El advenimiento, después del cataclismo, de una era verdaderamente cristiana, más pura, más piadosa, y por tanto, de mayor comunicación con Dios 5 .

En 1928 ya aparecen nítidamente definidos dos fundamentos básicos de la filosofía que guiará su trayectoria pedagógica hasta la muerte: el misticismo de la acción y una moral pragmática maleable a su misión pedagógico-apostólica. Estos principios se delinean por ella misma en la carta escrita desde Columbia, unos meses antes, a su íntima amiga María Baeza:

Yo tengo que emplear, mi querida María, en mi labor constructiva procedimientos puros de una moral que a las derechas le huele a azufre y a las izquierdas les parece poco revolucionaria. Es el destino de toda labor de esta índole: mantenerse

Morla Lynch, C.: En España con Federico García Lorca, Madrid, Aguilar, 1957.

Ibid., p. 94, «Me siento casi plenamente feliz pues me ha tocado en suerte vivir la existencia que yo habría elegido».

Díez Fernández, P.: «La dictadura de Primo de Rivera. Una oportunidad para la mujer», Espacio, tiempo y forma, Serie v, unED, Historia contemporánea, t. 17 (2005), pp. 175-190.

4 VÁzquez RamiL, R. Mujeres y educación en la España contemporánea. La Institución Libre de Enseñanza y la Residencia de Señoritas de Madrid, Madrid, Akal, 2012.

5 Morla Lynch, C.: op. cit., p. 96. 
EN LA FRONTERA: SEÑAS DE IDENTIDAD DE LA LABOR PEDAGÓGICA HISPANO-AMERICANISTA EN MARÍA DE MAEZTU (I924-I936) ELVIRA M. MELIÁN

en la frontera, en aquella raza espiritual donde vienen a estrellarse unas veces y a fundirse otras las enconadas pasiones de ambos bandos ${ }^{6}$.

Desde un notorio europeísmo inicial, íntimamente ligado a su vinculación ideológica con la $\mathrm{JAE}^{7}$, su particular frontera se irá desplazando tras los pasos de su hermano Ramiro de Maeztu ${ }^{8}$, del que afirmará en 1927 «al fin no ha podido más y ha pasado el Rubicón»9, ante su tránsito a un tradicionalismo católico-hispanista cercano al fascismo ${ }^{\text {Io }}$. Pese a ello, María se mantendrá como rostro femenino de la JAE y todopoderosa directora de la Residencia de Señoritas desde su creación en I9I5 hasta 1936, gracias a una camaleónica capacidad para armonizar mundos difícilmente ensamblables y transitar entre notables vaivenes sociopolíticos ${ }^{\text {II }}$. Esta dilatada permanencia, junto a su ambición personal y excepcional capacidad de trabajo ${ }^{12}$, explica la fecundidad de su obra dentro de la $\mathrm{JAE}^{13}$. Pero el coste de su

6 Fundación Ortega y Gasset: Correspondencia de María de Maeztu (cmm), Archivo de la Residencia de Señoritas (ARs). La voz de María de Maeztu utilizada en este trabajo es -fundamental, pero no exclusivamente- su correspondencia con María Baeza, por motivos de espacio, utilidad para su objetivo y estudios personales previos. Se enumera como Maeztu a receptos y fecha. Cuando la voz es de otras personas, etiquetadas como «Notables» en el Archivo la signatura será caja/carpeta/ página o caja/fecha, según catalogación. En este caso es CMM/ARs/ Maeztu a Baeza (04/04/1927).

La biografía intelectual de María de Maeztu admite cientos de trabajos sobre su labor pedagógica. La interacción con la JAE o Instituciones dependientes se iniciará como becaria (Londres, I908; Bruselas, Amberes y Zúrich, I910, y Maburgo, I9I2-I3). Posteriormente será designada para cuatro cargos principales: directora de la Residencia de Señoritas, I916; presidenta del Instituto Internacional de Señoritas, 1917; directora del grupo de niños de la Sección Preparatoria del Instituto-Escuela, I9I8, y, finalmente, vocal de la JAE en I928, siendo la única mujer que ocupó este cargo. Ya en su madurez, entre I9I9 y I929 la JAE le financiará varias estancias EE. UU. y América del Sur. Sobre la amplia vinculación entre Maeztu y la JAE consultar MARÍn Eced, T.: La renovación pedagógica en España (1907-1936). Los pensionados en pedagogía por la Junta de Ampliación de estudios, Madrid, Consejo Superior de Investigaciones Científicas, 1989; de la misma autora: Innovadores de la educación en España (Becarios de la JAE), Castilla-La Mancha, Universidad de Castilla-La Mancha, 1991. Sobre su vinculación con el Instituto Internacional consultar Zulueta, C.: Misioneras, feministas, educadoras, Madrid, Castalia, 1984; sobre su proximidad a Ortega y a Natorp y su Pedagogía social, ver Melián, E. M.: «María de Maeztu Withney y Sofía Novoa Ortiz (I9r9-I936), cultivar la salud, cultivar el espíritu, cultivar la lealtad», Circunstancia: Revista de Ciencias Sociales del Instituto Universitario de Investigación Ortega y Gasset, I4 (2007). Dicho artículo puede consultarse en la web de la fundación Ortega y Gasset www.fog.es/circunstancia/numeror4/art7.

8 Maeztu, M.: Antología del siglo XX. Prosistas españoles, semblanzas y comentarios, Madrid, Espasa Calpe, 1964 .

9 CMM/ARs/Maeztu a Baeza (04/04/1927).

ro Rivera García, A.: «Ramiro de Maeztu: biografía intelectual del caballero de la Hispanidad», Analecta Malacitana, xxIV (200I), pp. 603-607.

" De Zulueta, C. y Moreno, A.: La Residencia de Señoritas, Madrid, csic, I993; PérezVillanueva Tovar, I.: La Residencia de Estudiantes I9I0-1936. Grupo Universitario y Residencia de Señoritas, Madrid, CSIC, 20 II.

${ }_{12} \mathrm{CMM} / \mathrm{ARS} / \mathrm{Maeztu}$ a Calandre (06/07/1920). Con motivo del ingreso de Luis Calandre Ibáñez, amigo y prestigioso médico, en la Real Academia de Medicina, le escribe «cuánto le envidio, a ver si me ayuda para que yo pueda sentarme pronto en aquellos escaños».

${ }_{13}$ La biografía más completa sobre María de Maeztu sigue siendo la de Pérez-Villanueva Tovar, I.: Maria de Maeztu: una mujer en el reformismo educativo español, Madrid, UnED, I989. Puede consultarse también Fructuoso Ruiz, M. C.: María de Maeztu Withney, una victoriana ilustre, Álava, Eginzak, 1998. 
empresa en términos físicos, psíquicos y éticos no será banal: entre 1924 y I936, periodo recogido en este trabajo, su correspondencia evidencia una íntima soledad, acentuada por ideas de acoso oficial, y la permanente necesidad de rediseñar un entramado ético-moral donde asentar su dúctil pragmatismo vital.

El objetivo de este trabajo es acercarnos a la fecunda labor de María de Maeztu, como integrante de un movimiento pedagógico hispanista femenino-cristiano poco investigado, que alcanzó su apogeo en el primer tercio del siglo xx. Utilizando sus cartas, escritos y ensayos analizaremos, por un lado, su evolución ideológica y su ambivalente relación con la JAE durante este periodo, universos ambos imprescindibles para interpretar su aportación personal al mismo. Y, por otro, el determinante papel que ocupó en su compromiso hispano-americanista la urdimbre tejida con otras figuras tan notorias de esta corriente como fueron Gabriela Mistral o Blanca de los Ríos.

En este vínculo con una «comunidad que ha de realizarse en el reino del espíritu porque la Patria no está formada por el territorio que los hombres habitan, sino por el espíritu que los ensalza en la conciencia universal» ${ }^{14}$, María dejó profundas huellas de su íntima visión pedagógica. Más allá de mudanzas temporales, los documentos examinados encierran la inmutable esencia de una vocación docente que, en María de Maeztu, se unificó con la propia vida.

\section{Relaciones con la Junta para Ampliación de Estudios e Investigaciones Científicas (1924-1936)}

María de Maeztu alcanza el esplendor de su tarea pedagógica traspasado el umbral de los 40 años. Para entonces la Residencia de Señoritas es un éxito, como evidencian el significativo aumento de alumnado -que obligará a la creación de varias «Casas»-, la ampliación de la oferta educativa o su interacción con los fo-

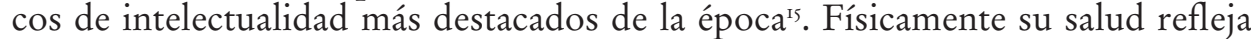
las secuelas de años de trabajo agotador que intentará enmascarar toda su vida ${ }^{16}$ y, dos años antes de conocer a Morla Lynch, la ha obligado a permanecer varios meses en Berna convaleciente de un proceso tiroideo que oculta, por temor a que la obliguen a dimitir ${ }^{17}$.

El éxito en la vida requiere muchas cosas... muchas. Los que más o menos lo hemos tenido unas horas sabemos bien de qué hilos tan sutiles se compone. Pero requiere más que nada, y como condición previa, salud que es: fuerza energía capacidad en la lucha para no ser vencidos por los miserables; y cuando no se tiene hay que fingirla ${ }^{18}$.

${ }^{14}$ Sin Firma: «En el paraninfo de la Universidad Central se inauguró ayer solemnemente la cátedra Ramiro de Maeztu», $A B C, 30-\mathrm{I}-1947$, p. II.

is Pérez-Villanueva Tovar, I.: 20II, op. cit.

$16 \mathrm{CMM} / \mathrm{ARs} / \mathrm{Maeztu}$ a Baeza (sin día/o9/I925), «Empecé a trabajar a los 17 años y tengo 43. Quiere decir 26 años de una labor sin descanso fundando escuelas, instituciones, corriendo España de punta a punta en un tren de tercera para predicar no sé qué vago ensueño que como ensueño y como vago no tenía el más menudo valor... pero la llama encendida de mis años jóvenes se apagaba».

${ }^{17}$ Melián, E. M.: 2007, op. cit.

I8 CMM/ARs/Maeztu a Baeza (sin día /og/r925). 
EN LA FRONTERA: SEÑAS DE IDENTIDAD DE LA LABOR PEDAGÓGICA HISPANO-AMERICANISTA EN MARÍA DE MAEZTU (I924-I936) ELVIRA M. MELIÁN

La larga epístola enviada a María Baeza desde Suiza anuncia la que será recurrente discrepancia entre la privilegiada posición objetiva que sustenta hasta 1936, y un cierto victimismo subjetivo, reagudizado en periodos de transición política ${ }^{19}$.

Yo tenía miedo de que al crecer mi obra creciera en la misma proporción el número de enemigos o la fuerza de los pocos que tengo y que no habrían de perdonarme el delito de hacer una obra social en España [...] y así fue, durante esos seis meses no me han dejado vivir, me han matado. No, todavía no, pero han hecho lo posible ${ }^{20}$.

En 1925 sus «enemigos» se encuentran dentro de la JAE, a los que, siempre según su correspondencia, se ha enfrentado meses atrás, en la persona de su secretario, por una deuda de dos años de antigüedad hacia la Residencia de Señoritas ${ }^{21}$. Como respuesta se le ha sugerido que dimita acusándola de «injuriar a la Junta [...]» porque «en España siempre es una injuria pedir que se paguen las deudas» ${ }^{22}$. Dramática declaración que contrasta con las memorias de la JAE de estos años donde observamos que, amén de recibir financiación para diferentes conceptos de la Residencia, es pensionada para Argentina/Uruguay (1926) y Estados Unidos $(1927)^{23}$. De ambos pensionados volverá a Madrid con una terrible angustia por «acoplarse a una vida tan áspera y tan dura como lo es la española» ${ }^{24} \mathrm{y}$, en I927, temiendo «lo que me espera a mi regreso de esos miserables que antes no me dejaban vivir y ahora más que nunca se creerán en el deber de posicionarse como enemigos declarados ${ }^{2}{ }^{2}$. Por la fecha deducimos que los miserables son miembros de la JAE y de la intelectualidad de izquierdas, que tan pronto como en 1924 han firmado un manifiesto ${ }^{26}$ contra el régimen de Primo de Rivera, del que ella será colaboracionista ${ }^{27}$.

Entre 1928 y 1930, caída del dictador, María de Maeztu ocupa una poderosa posición como miembro de la junta directiva de la JAE, sustentada en parte sobre la significación de Ramiro de Maeztu ${ }^{28}$. Serán años de álgido trabajo en favor de la Residencia, en los que sus posiciones ideológicas se escoran desde un regeneracionismo

19 El estudio de esta íntima amistad, que permite un sensible acercamiento a la figura de María de Maeztu, ha sido realizado por mí con anterioridad, Melián, E. M.: «Rastro de nubes: María Martos de Baeza y su mundo", Arenal: Revista de Historia de Mujeres, 8 (200I), pp. 379-388; ver también Ribagorda, A.: «Una historia en la penumbra: las intelectuales de la Residencia de Señoritas», Sistema, I88 (2005), pp. 54-6I.

$20 \mathrm{CMM} / \mathrm{ARS} / \mathrm{Maeztu}$ a Baeza (sin día /og/r925).

${ }_{21}$ Ibid. Esta afirmación, no contrastada, la hace a María Baeza en su carta arriba señalada.

${ }_{22}$ Ibid. Según escribe ha respondido con un órdago, «una carta valiente y enérgica que ni por una orden de la más alta representación del país abandonaría mi puesto» pues -sigue confesando a María Baeza- «sólo las I5o alumnas que viven conmigo podrían echarme. Mientras ellas estén junto a mí el resto no vale nada».

${ }_{23}$ Memorias de la JAE, http://archivojae.edaddeplata.org/jae_app.También es útil el trabajo de MARTÍn ECED, T.: 199I, op. cit.

24 CMM/ARs/Maeztu a María Chinchilla (22/II/1926).

25 CMM/ARs/Maeztu a Baeza (16/05/1927).

26 Ortega, Menéndez Pidal y Unamuno, tres de los maestros de María, aparecen entre los firmantes del manifiesto reseñado.

${ }_{27}$ Ribagorda, A.: «El drama de los liberales», Claves de Razón Práctica, I60 (2006), pp. 58-66.

28 Martín Eced, T.: 199i, op. cit. 
empírico deudor de Ortega ${ }^{29}$ hacia la derecha tradicionalista, con abierto apoyo a un catolicismo monárquico personificado en el Estado. Conforme su «obra» «que es, usted lo sabe, más querida que un hijo, vive el momento pleno de su éxito»30, cualquiera que la amenace será considerado como hostil. En I93I, realizada la transición a la II República escribirá,

Yo he sacrificado a mis dos grandes amores: mi obra y Ramiro, todo es que ahora se llama popularidad. No me pesa; hice en cada instante lo que tenía que hacer porque no era libre para arrojar mi obra -que es toda mi vida- por la ventana. ¡Cómo ha de ser! Cuando pasen unos meses todo volverá a su lugar, es decir todo lo que merezca que vuelva ${ }^{35}$.

No se equivoca. La República «pondera su labor»32 y, en marzo de I931, cuando se reabre el Ateneo de Madrid, clausurado en plena sublevación militar, la encontramos, junto a Blanca de los Ríos, entre el grupo de intelectuales y artistas convocados como «las más altas personalidades de la vida científica, sin excluir la política» para "disertar de los palpitantes problemas de España»33. Hasta 1936 su Residencia recibe significativas ayudas - «luego se dirá que debíamos algo a la monarquía»-34, y el inicio de la Guerra Civil la encuentra felizmente incorporada a la docencia en las cátedras de Pedagogía e Historia de la Pedagogía de la Universidad Central, al ser nombrado Luis de Zulueta, su titular, embajador de España en la Santa Sede ${ }^{35}$.

Durante los años previos a la dictadura de Primo de Rivera María de Maeztu lideró, en unión con otras relevantes mujeres, pioneras iniciativas intelectuales y cívicas, cuyo análisis sobrepasa los límites de este trabajo ${ }^{36}$. El advenimiento de la República dispersa a estas amigas y María, intelectualmente lejana a la mayoría de sus contemporáneas ${ }^{37}$, vive dolorosamente esta forzosa separación. Aunque en 1932 escribe a Baeza, embajadora en Chile, «todo bien, muy bien [...] se lo digo

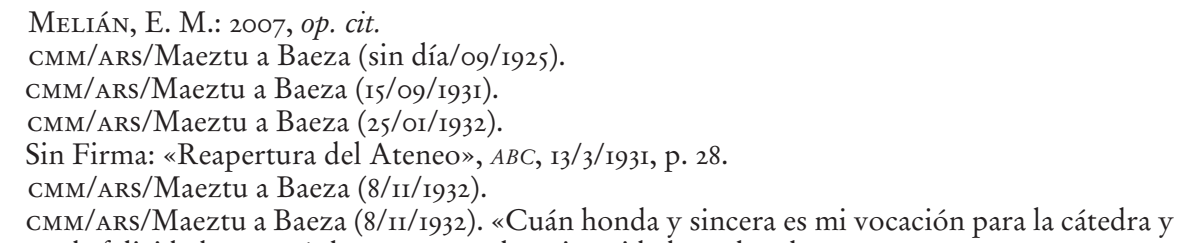

${ }_{36}$ En este periodo destacan entre otros la creación del Lyceum Club, que sobrevivirá hasta el inicio de la Guerra Civil. Sobre el tema ver Melián, E. M.: 20oI, op. cit. y Melián, E. M.: «Memorias de la Penumbra: Carmen Baroja, Zenobia Camprubí y Elena Fortún, mujeres en la penumbra del 98», Boletín Institución Libre de Enseñanza, 5 (2005), pp. 19-39. En este artículo se analiza la correspondencia y amistad entre María de Maeztu y mujeres como Carmen Baroja, Elena Fortún o Zenobia Camprubí, que abrieron vías impensables para la mujer en éste y otros ámbitos culturales, laicos y femeninos. Sobre este asociacionismo femenino ver DíEz Fernández, P.: op. cit. Y son especialmente cercanas, dado que fueron escritas por una de sus ejecutoras, las memorias de BAROJA, C.: Recuerdos de una mujer de la generación del 98 , Madrid, Tusquets, 1998.

37 CMM/ARs/Maeztu a Baeza (sin día/ı2/1924), «es muy difícil coincidir, muy difícil; más aún entre mujeres no porque la coincidencia no sea posible sino porque falta una base de contenido común». 
EN LA FRONTERA: SEÑAS DE IDENTIDAD DE LA LABOR PEDAGÓGICA HISPANO-AMERICANISTA EN MARÍA DE MAEZTU (I924-I936) ELVIRA M. MELIÁN

María, para que se haga una idea de lo bien que se presentan las cosas» ${ }^{38}$, su soledad se acentúa ante el temor a represalias políticas - «no quedan en pie más que los que caminan muy muy hacia la izquierda»-39, y la animadversión que percibe hacia su persona, tras la caída del Régimen - «yo no sospechaba que me tuviesen tal odio... pues no les hice más que bien»-. En sus palabras:

Me encuentro en una soledad interior como nunca me he sentido en la vida [...]. Ahora al perder a Ramiro, quiero decir, al perder todo lo que durante muchos años ha representado su apoyo como dice la gente, su sombra, sé lo que es caminar sola, trágicamente sola, en un mundo, como el actual donde por todas partes brotan espinas ${ }^{\circ}$.

Soledad que siempre encontrará en el trabajo su más eficaz consuelo.

\section{La íntima filosofía de una vocación pedagógico-apostólica}

María de Maeztu es una muestra de la supremacía de la voluntad - «acción» sobre cualquier otra fuerza ${ }^{41}$ y defiende que al hombre occidental no le basta ser, tiene el imperativo de hacer ${ }^{42}$. En coherencia practicará, como Teresa de Jesús, «un amor que no se detiene en la contemplación y busca un cauce a su anhelo en las obras humanas que funda [...] y mientras levanta el corazón a Dios, en oración fervorosa, pone con manos certeras y actividad continuada los ladrillos de sus fundaciones» ${ }^{43}$. Para ella la vocación pedagógica es una llamada íntima, inajenable e insustituible por otros y, parafraseando a Wells, afirma que «la escuela debe ser para el maestro el altar donde ofrenda a Dios su vida»44. Misión para la que propone intensificar el trabajo intelectual, por un lado, y comunicar el aliento místico-pedagógico en círculos excéntricos, por otro ${ }^{45}$.

En esta creencia podemos integrar su identificación con Teresa de Jesús, sobre la cual disertará durante un ciclo de conferencias auspiciado por la JAE en 1926 en Argentina y Uruguay, brindándonos valiosos testimonios de su idiosincrasia. Su

\footnotetext{
CMM/ARs/Maeztu a Baeza (8/II/1932).

CMm/Ars/Maeztu a Baeza (I5/o9/193i).

CMM/ARs/Maeztu a Baeza ( 8/II/I932)
}

${ }_{41} \mathrm{CMM} / \mathrm{Ars} /$ Maeztu a Unamuno (sin día/o2/192I). En febrero de I92I rechaza una invitación de Unamuno para dar unas charlas en el Ateneo salmantino en estos términos: «Este año mi querido don Miguel tengo mi trabajo tan mal arreglado que no me deja a solas para pensar. No es que tenga todas las horas tomadas pero las que me quedan libres no me abandona la preocupación de mil detalles menudos y constantes que me impiden leer y pensar con reposo. Pero muy en breve podré arreglar mi vida de otra manera y entonces volveré a mi trabajo intelectual y a la propaganda de mis ideas por medio de conferencias».

${ }_{42}$ Maeztu, M.: El problema de la ética. La enseñanza de la moral, Buenos Aires, Universidad de Buenos Aires, I938.

43 Maeztu, M.: Conferencia pronunciada el 24 de julio de 1926 por la doctora María de Maeztu, Centro Gallego de Montevideo. Tema: Historia de la cultura femenina en España. Existe un folleto de la misma editado por el propio centro, 1926.
${ }_{44}$ Maeztu, M.: 1938, op. cit., p. I04.
45 Ibid., p. I83. 
EN LA FRONTERA: SEÑAS DE IDENTIDAD DE LA LABOR PEDAGÓGICA HISPANO-AMERICANISTA EN MARÍA DE MAEZTU (I924-I936) ELVIRA M. MELIÁN

anfitriona uruguaya, la preeminente poeta Juana de Ibarbourou, la presenta al público del Centro Gallego de Montevideo, asegurando que es «de la pasta de las mujeres de Castilla en el siglo xvi [...]» pues «funda escuelas como Santa Teresa fundaba conventos. Posee el espíritu fuerte y ejecutivo de la santa doctrina de Ávila. Y tiene como ella la sabiduría amable, el valor sonriente, el optimismo, el tesón y la fe» ${ }^{46}$. En palabras de María la carmelita «si hubiera vivido en nuestros días hubiera fundado hospitales, escuelas, universidades». No en vano,

En la obra, al realizarla, se afirmaron de una vez para siempre las cualidades de Teresa: voluntad inflexible, irresistible fuerza de seducción, diplomacia para manejar a los amigos y desarmar a los enemigos, fe inquebrantable en los destinos de su raza, seguridad de vencer, y todo ello sin perder jamás la gracia femenina, la sonrisa, el gesto, la alegría ${ }^{47}$.

El libro El problema de la ética, la enseñanza de la moral, escrito durante el exilio bonaerense en 1938, compendia un pensamiento pedagógico que coloca a la religión, «elemento situado en la esfera del sentimiento de lo infinito que es a su vez el seno materno de la conciencia» ${ }^{4}$, como pivote de la educación. En él María establece explícitamente la conexión entre moral y religión, a través de la cadena lineal moral-deber-regla-obligación-disciplina-autoridad-admiración-religión. Estas líneas maestras de actuación pedagógica se dibujan ya en el ensayo que escribe en I925 sobre la educación en el Instituto Escuela, donde afirma que «en los primeros años de la formación humana -la religión- es la expresión más elevada del sentimiento, con un título idéntico o superior al de la ciencia, la moral y el arte»49. En una identificación entre moral y disciplina del cuerpo social la maestra subordina, desde la más tierna infancia, el fomento del pensamiento independiente, la libertad, la responsabilidad y el espíritu crítico a la idea matriz de que «la libertad en sentido pleno no es más que sumisión a la ley»". Para ella, «la obediencia que la disciplina exige no significa negación de la voluntad propia; consiste simplemente en someterse a la voluntad del guía porque es el que mejor conoce el camino» ${ }^{\Upsilon 1}$. Cuando el éxito de la Residencia de Señoritas, su particular fundación, requiera que sea dividida en Casas para su gobierno, María escribe unos estatutos que inciden en el atributo espiritual de su misión pedagógica. La tutoría de las jefas de grupo debe operar fundamentalmente sobre la vida moral de las alumnas, exhortadas a aplicar las reglas de moralidad a través de «conquistar la confianza de las jóvenes empleando resortes de persuasión y autoridad que emana del propio prestigio personal a fin de que las alumnas se sientan felices

${ }_{46}$ Maeztu, M.: Conferencia pronunciada el 24 de julio de 1926 , op. cit., p. 7.

47 Ibid., p. 4.

${ }_{48}$ Maeztu, M.: 1938, op. cit., p. 224.

49 JAE: El Instituto Escuela de Segunda Enseñanza de Madrid (organización, métodos, resultados), Madrid, Lumen, 1925, p. 39. Se trata de una publicación donde colabora en el apartado de la Sección Preparatoria, capítulos de Enseñanza de la Religión, Enseñanza de la Geografía y Enseñanza de la Lengua Castellana.

so Ibid., p. 33

st MaezTu, M.: 1938, op. cit., p. 134 . 
EN LA FRONTERA: SEÑAS DE IDENTIDAD DE LA LABOR PEDAGÓGICA HISPANO-AMERICANISTA EN MARÍA DE MAEZTU (I924-I936)

ELVIRA M. MELIÁN

y se confíen a su dirección». Cumpliendo, eso sí, «las Reglas del Libro Azuls, pues toda perturbación en la Ley engendra una relajación de hábito que estorba el cumplimiento del deber» ${ }^{3}$. Y, sobre cualquier otra consideración, exige de sus colaboradoras «fidelidad absoluta a la persona que ha fundado y preside la Obra» 54 .

Perdida la dirección de la Residencia al inicio de la Guerra Civil, y sufriendo un doloroso exilio en Argentina, María continuará su lucha a favor de la educación femenina hasta su último aliento ${ }^{5}$. La impuesta desubicación geográfica la conduce a proyectar esta vocación sobre la «congregación de pueblos libres que tienen una comunidad de idioma, de religión; un mismo estilo de vida y, en cierto sentido, una análoga tarea a realizar» ${ }^{56}$. Una tarea que podemos integrar en años de efervescencia americanista simbólicamente enmarcados entre el «concepto de afinidad cultural, lingüística e histórica» de Unamuno como precursor, y la «propuesta de la hispanidad como comunidad espiritual vinculada a una interpretación católico-tradicionalista de la historia de España» de Ramiro de Maeztu, como epílogo ${ }^{57}$. Empresa a la que no será ajena su amistad con otras dos fervientes hispano-americanistas, Blanca de los Ríos y, en especial, la famosa poeta Gabriela Mistral.

\section{María de Maeztu, Gabriela Mistral y Blanca de los Ríos, o el semblante humano del hispano-americanismo femenino cristiano del primer tercio del siglo $\mathrm{XX}$}

María de Maeztu conoce a Gabriela Mistral en diciembre en 1924 cuando la ya famosa poeta y dos compañeras se alojan en la Residencia de Señoritas, durante su estancia en España. El primer domingo de diciembre a la caída la noche se celebra en la Casa, Fortuni 53, un agasajo por parte de la anfitriona, al que acuden «más de 200 personas: el cuerpo diplomático, escritores y artistas de primera fila, en fin lo mejor de Madrid ${ }^{58}$. Para la organizadora tendrá mucho más valor la entrañable ceremonia celebrada la noche previa en compañía de sus estudiantes. Bien vale reproducir aquí sus palabras recogidas en su correspondencia con Martos de Baezas,

52 Era el libro de reglas de la Residencia de Señoritas. Si las directoras de cada Casa querían cambiar cualquier regla deberían hacer sus sugestiones a la directora, que las consideraría o no para el siguiente año académico.

53 Sobre el manejo de las Casas consultar Melián, E. M.: 2007, op. cit.

54 Las obligaciones de las responsables de las Casas fueron muy elevadas. Su madre Juana Whitney afirmaría que "María no se casa porque lleva su profesión de maestra como si fuese un sacerdocio» y, en cierto modo, debió exigir de sus alumnas una entrega similar.

"s Altuna de Martina, A.: «María de Maeztu: tras sus pasos por Argentina», en La mujer vasca en el exilio, País Vasco, Saturrarani, Hamaika Bide, 2007, pp. 383-407.

56 Del Corral, E.: «Hay que vivir y trabajar en América para conocerla», $A B C, 2 \mathrm{I} / 2 / 1945$, p. 19.

57 EgIdo LEón, Á.: «La hispanidad en el pensamiento reaccionario español de los años treinta», Hispania, Madrid, 53, n. ${ }^{\text {I } 84}$ (1993), pp. 65I-673.

58 Entre los invitados a este evento destacan Juan Ramón Jiménez y Zenobia Camprubí, Azorín, Eduardo Marquina, Eugenio D’Ors, Pérez de Ayala, Alberto Jiménez Fraud o José Rodríguez Cariacedo, entre otros.

59 Melián, E. M.: 200I, op. cit. 
Ahora tenemos en la casa a Gabriela Mistral: mujer magnífica, muy superior en su personalidad compleja y rica a su obra literaria. El sábado por la noche le rendimos un homenaje aquí, en la biblioteca, nuestro homenaje dentro de la mayor intimidad. No invitamos más que a Ella ${ }^{60}$. Yo hice la presentación y leí algunas de sus poesías y "La oración de la maestra»; y ella de una manera espontánea y admirable hizo un comentario a la oración. Comentario tan lleno de fervor religioso que nos transportaba a un mundo lejano distinto. Las chicas la recibieron como no han recibido hasta ahora a nadie. Cuando ella se levantó a hablar fue un momento de intensa emoción ver a las 170 alumnas, puestas en pie, tributándole una ovación que duró unos minutos ${ }^{61}$.

Días más tarde durante una comida organizada por el PEN club en el Hotel Savoy en honor a la poeta ${ }^{62}$, María la introduce al auditorio evocando «la honda huella en el alma de nuestras muchachas jóvenes, que al escucharla explicar su comentario a la "Oración de la Maestra" han sentido el contacto de su llama encendida y el atractivo de esa emanación de santidad que sólo los seres excepcionales llegan a sentir» ${ }^{63}$. Emanación de santidad que brilla en un discurso donde destaca sobremanera «que en ella (Gabriela Mistral) es su vocación religiosa lo más encendido de su personalidad». Esa parece ser la imagen que de la poeta tienen los que la conocen, austera, severa, una «religiosa laica». Aunque Gabriela es una joven de 35 años que ha publicado únicamente un poemario, y no será galardonada con el premio Nobel hasta 1945, la sexagenaria Blanca de los Ríos, que ese mismo año ha recibido de Alfonso XII la Gran Cruz de la Orden Civil ${ }^{64}$, parece compartir la admiración de María de Maeztu hacia la chilena ${ }^{65}$. Así se deduce de la edulcorada nota que dirige a Gabriela, asumimos entregada en la reunión del Savoy, y que quedará en la Residencia tras su partida a América ${ }^{66}$.

Las tres mujeres comparten su afinidad por la idea de Iberoamérica, su espíritu pedagógico y un profundo cristianismo. Por su parte, las dos españolas tienen en nuestras fronteras una notoriedad importante, aunque María de Maeztu muestra hacia Blanca gran respeto en razón de su edad y origen. Perteneciente por

60 Isabel Oyarzabal de Palencia. La correspondencia con María de esta importante protagonista de iniciativas como el Lyceum Club puede consultarse en CMM/ARs/Oyarzabal a Maeztu (40/53/pp. I-27).

6r CMM/ARs/ Maeztu a Baeza (sin fecha/I2/I924).

62 El i6 de enero de i924.

${ }_{63}$ Sin Firma: «Homenaje a la poetisa chilena Gabriela Mistral», El Sol, I7-I2-I924, p. 2.

${ }_{6}$ González López, M. A.: Aproximación a la obra literaria y periodística de Blanca de los Ríos, Madrid, Fundación Universitaria Española, 200 .

${ }_{65}$ De los Ríos solicita personalmente una fotografía a Gabriela Mistral, que esta dejará a María de Maeztu, la cual, según se desprende de su correspondencia personal, la envía a Victoriano Fernández Alcázar, director del Magisterio Español para su publicación.

66 «A Gabriela Mistral que ha sabido modelar el ánfora grácil de la forma con los dedos enfebrecidos de amor y amasarla -según su decir- "con el lodo de las hojas secas”, para que diese al beber "el olor de los otoños, que es el perfume mismo de su corazón”; y al verter en ellas las lágrimas de su trágico dolor mezcladas con luces columbradas desde la cima de su espíritu, la ha llenado del néctar que no se agota, del néctar de llanto y de luz de la mística poesía, que pega sabor de tristeza y fe y de infinito a todos los paladares; Gabriela Mistral, que nos trae palpitantes en sus versos el alma de la América hermana, envío con estas letras una efusión de afecto aún más íntima que la paternidad (de raza, la de la santa fraternidad del arte, "cuya misión es -como ella-dice embellecerlo todo de un inmensa misericordia”». CMM/ARs/De los Ríos a Mistral, 2I/(I6/I2/I924). 
EN LA FRONTERA: SEÑAS DE IDENTIDAD DE LA LABOR PEDAGÓGICA HISPANO-AMERICANISTA EN MARÍA DE MAEZTU (I924-I936) ELVIRA M. MELIÁN

familia a la élite intelectual sevillana, su matrimonio con el conocido arquitecto y arqueólogo madrileño Vicente Lampérez y Romea formaliza esta privilegiada posición original. Poeta, narradora y sobre todo erudita del Siglo de Oro, Blanca es socia de número del Ateneo desde i895, privilegio únicamente compartido por Pardo Bazán y Carmen de Burgos ${ }^{67}$.

El Ateneo, nacido en I820, es el centro más influyente sobre la opinión pública en Madrid, y un foro donde prestigiosas personalidades debaten, entre otros muchos temas, la ambigua situación femenina durante el primer tercio del siglo xx. Blanca de los Ríos y María de Maeztu participarán de este foro, representando dos prototipos de emancipación femenina, que asumirán con coherencia en su trayectoria vital. La sutileza de estas diferencias se aprecia en una entrevista realizada en 1917 por Gregorio -María- Martínez Sierra para el libro titulado La mujer moderna ${ }^{68}$. Mientras Maeztu se declarara abiertamente feminista (emancipación económica, emancipación intelectual, participación en las leyes, trabajo cultural) y apoya a los grupos así llamados, Blanca reprueba este concepto. En contraste ambas: I, defienden la existencia de diferencias cualitativas entre mujer y hombre y niegan el antagonismo entre feminidad y feminismo instituido por las feministas; 2, son partidarias de reformar las leyes, defendiendo la no inferioridad intelectual de la mujer, y 3, preconizan el valor de la educación como medio para la igualdad. Si bien María considera la educación un ejercicio principalmente de acción (la mujer es la principal enemiga de la mujer) y Blanca se muestra más propensa a una educación de tipo evolución sin revolución. No en vano en un ciclo de conferencias sobre la mujer, impartidas en el Ateneo en I9I2, Blanca escoge disertar sobre «La mujer y la mística» y María sobre «La mujer y la escuela» ${ }^{6}$.

El tratamiento de la figura de Teresa de Jesús, otra constante en sus vidas, es igualmente significativo ${ }^{70}$. Las españolas coincidían en considerarla como primera mujer que aunó en nuestro país la vida activa con la contemplativa, e incluso en la visión de Blanca aupándola a «ideal místico de la raza» y «renovadora espiritual en su calidad de escritora por excelencia» ${ }^{71}$. Pero si para Blanca de los Ríos «con Santa Teresa la mujer se incorporó triunfalmente a la vida intelectual del mundo ${ }^{7^{2}}$, para María de Maeztu el axioma decimonónico se ha transformado en la visión de la carmelita como un modelo de educadora y reformadora social. Como mujer de acción María de Maeztu creará instituciones simbólicas para el desarrollo femeninista del primer tercio del siglo xx como el Lyceum Club ${ }^{73}, \mathrm{y}$ se encontrará en el origen de las principales agrupaciones feministas de la época

${ }^{67}$ Abellán, J. L.: El Ateneo de Madrid. Historia, Política, Cultura, Teosofía, Madrid, ed. La Librería, 2006.

68 Martínez Sierra, G.: La mujer Moderna, Madrid, ed. Estrella, i92o.

69 Sin Firma: «Aspectos sobre la mujer», La Correspondencia de España, 5/1/1912, p. 5.

70 Las tres serán defensoras de la biografía individual, sustento del concepto de «intrahistoria» unamuniano.

${ }_{71}$ De Los Ríos, B.: Influjo de la mística de Santa Teresa singularmente sobre nuestro grande arte nacional. Conferencia leída en la Real Academia de Jurisprudencia el 20 de febrero de i913, Madrid, Unión de Damas Españolas, i913.

\footnotetext{
72 Ibid., p. 24.

73 Melián, E. M.: 2005, op. cit.
} 
entre las que destacan la Asociación Nacional de Mujeres Españolas (ANME) ${ }^{74}$ o la Federación Internacional de Mujeres Universitarias (FIMU) ${ }^{75}$. Como mujer contemplativa, Blanca de los Ríos apoyará movimientos ligados a la derecha católica tradicional del tipo de la Unión de Damas del Sagrado Corazón de Jesús o la Unión de Damas Españolas ${ }^{76}$.

Por su parte Gabriela Mistral comparte con esta última la admiración a la santa Teresa más mística, y muestra gran similitud de posiciones respecto al feminismo. Así, en sendos artículos publicados de forma independiente por Mistral y De los Ríos el año de 1927, sin identificarse con un feminismo ortodoxo, participan de unos ideales de libertad e igualdad en base a la educación y la equiparación moral ${ }^{77}$. De hecho se ha interpretado el texto de Blanca de los Ríos definiendo en santa Teresa un «anhelo vehemente de asociar nuestro sexo a la magna obra de Cristo» incluso de feminismo teresiano ${ }^{78}$. Recordemos que durante la República, y dentro de las derechas católicas, el teresianismo sería el movimiento más favorable a la equiparación política, social y general de la mujer en la vida pública ${ }^{79}$. Estas ideas se enmarcan en tímidas demandas sociales de derechos femeninos, y no sorprende que para las tres amigas la función suprema de la naturaleza femenina fuera la maternidad y criar-educar a los hijos desde un feminismo cristiano ${ }^{80}$. Curiosamente ninguna fue madre.

Cuando en febrero de 1925 Gabriela embarca desde La Coruña hacia Valparaíso, lleva consigo un profundo agradecimiento a la amiga vasca ${ }^{81}$, y así lo expone en el poema «Salutación», leído en el evento del PEN Club y publicado en El Sol el 17 de diciembre de $1924^{82}$. Las tres mujeres mantendrán a partir de entonces una relación intensa y desigual, como desiguales son sus personalidades que, en palabras de Blanca de los Ríos, tienen en común el «sentir arder en el alma el ascua

${ }_{74}$ Creada por María Espinosa de los Monteros en i9ı8. Ver Matilla Quiza, M. J.: «María Lejárraga y el asociacionismo femenino (1900-1936)», en AguIlera SASTRE, Juan (coord.): María Martínez Sierra y la República: Ilusión y compromiso: II Jornadas sobre María Lejarraga, Logroño 23-25 de octubre y 6-8 de diciembre 200I, 2002, pp. 83-IoI.

${ }^{75}$ MaIllard, M. L.: Asociación española de mujeres universitarias (1920-1990), Madrid, Instituto de la Mujer, I990.

${ }^{76}$ Núñez Pérez, M. G.: Madrid 1931. Mujeres entre la permanencia y el cambio, Madrid, Dirección General de la Mujer, I993.

77 Mistral, G.: La tierra tiene actitud de mujer (Pensamiento feminista, mujeres y oficios), Selección de Pedro Pablo Zegers, Santiago de Chile, ed. Rils, i998.

${ }_{78}$ Ezama Gil, Á.: «Ana Ozores y el modelo teresiano», ejemplaridad y escritura literaria, 2006. Este artículo se puede consultar en la Biblioteca Virtual Miguel de Cervantes. www.biblioteca.org.ar.

79 El artículo de Martínez Sánchez, R.: «Teresa de Jesús, apoteosis del feminismo», Mundo

Femenino, I/II/I935, pp. 8-9, sintetiza bien esta opinión. Ver también NúÑ̃ez PÉrEz, M. G.: op. cit.

8o Mistral, G.: «Feminismo: la opinión de Gabriela Mistral», La tierra tiene..., op. cit., p. 5I.

81 CMM/ARS/Mistral a Maeztu (2I/I5/ 1925, sin fecha).

82 Lago de Lapesa, P., «Una poesía olvidada de Gabriela Mistral».

«Palabra de mujer dijo de mi excelencia, garganta vasca donde conozco mi ascendencia.

Yo alabo, respondiendo, la anchura de su casa que tiene el buen calor de la profunda brasa.

La luz para gozar la cara de la amiga, Y el gran silencio para que duerma la fatiga.

Su casa es la virtud del aceite precioso, potente por la esencia y al tacto bondadoso.

La dueña abrió la casa, sin preguntarme nada.

¡Cómo el aceite, que es la piedad, sea loada!».

Texto disponible en www.InstitutoCervantes.com. 
EN LA FRONTERA: SEÑAS DE IDENTIDAD DE LA LABOR PEDAGÓGICA HISPANO-AMERICANISTA EN MARÍA DE MAEZTU (I924-I936)

ELVIRA M. MELIÁN

mística de la emoción estética hispano americana y aspirar a fundir en una sola inmensa vida la tradición romántica de España y su transmutación maravillosa en América» ${ }^{83}$. Demostración elocuente de este impulso de integración en las españolas son los trabajos de Blanca en Raza Española ${ }^{84}$, y la serie de conferencias impartidas por María de Maeztu en 1926 en Argentina y Uruguay, de las que hemos hablado ${ }^{85}$.

Entre 1927 y 1929 el destino las reúne de nuevo como parte de las trece mujeres que recibe en sus filas la Asamblea Nacional Consultiva de Primo de Rivera; Blanca por el mundo de la prensa y María por el mundo de la enseñanza ${ }^{86}$. Desde Buenos Aires Gabriela Mistral celebra esta oportunidad, en mayo de 1928:

Yo tengo que celebrar con honradez, y aunque no pongo a ningún fascismo gesto cariñoso, es acierto de sensatez y el buen atisbo de moralidad política que contiene la forma de representación femenina adoptada en España. Se ha liberado a María de Maeztu, a Doña Blanca de los Ríos y a sus compañeras, de la cosa sucia que es una batalla de urnas, con peroraciones en la plaza o el choclón y búsqueda mercenaria de electores. Sólo que se ha buscado cumplir con el feminismo en dosis infinitesimal, de una química bastante maliciosa. Una María de Maeztu representa en grande a su gremio, pero entidad tan vasta como la de maestra no se sacia de justicia con una sola diputada, aunque traiga sus cualidades ${ }^{87}$.

En septiembre de ese año se organiza la XII Conferencia de la Federación Internacional de Mujeres Universitarias ${ }^{88}$. Durante su estancia en la capital las delegadas se alojan en la Residencia de Señoritas, entre ellas Gabriela Mistral como delegada de Chile y Ecuador, aunque el país aún no se ha incorporado oficialmente a la organización ${ }^{89}$. En el banquete oficial, celebrado en el Hotel Palace, hablan

83 De los Ríos, B.: «Las mujeres españolas en I926», $A B C$, 2-I-I927, p. Io.

${ }_{4}$ Raza Española, revista creada y dirigida por Blanca de los Ríos entre i9ı8 y 1930. Ver GonZÁlez López, M. A.: op. cit.

85 Altuna de Martina, Á.: 2007, op. cit. Durante su viaje de 1926 a Argentina y Uruguay María dará clases sobre temas educativos, psicología pedagógica, metodología de la enseñanza e instituciones femeninas universitarias. Además, bajo el epígrafe de contribución española a la cultura pronunciará cuatro conferencias: Santa teresa de Jesús y sus Fundaciones; Sor María de Ágreda y su influencia en el reinado de Felipe IV; Sor Juana Inés de la cruz, y Escritoras españolas contemporáneas. Posiblemente se reencontraría con Gabriela Mistral en Buenos Aires, aunque no he podido confirmarlo.

86 Díez Fernández, P.: 2005, op. cit.

${ }_{87}$ Mistral, G.: «El voto femenino», en La tierra tiene..., op. cit., p. 63.

88 Preparan el evento, ampliamente recogido en la prensa, María de Maeztu -desde la vicepresidencia- y Clara Campoamor -desde la secretaría-, de la Juventud Universitaria Femenina (JUF), primera acepción de la Federación Española de Mujeres Universitarias. La Federación de mujeres Universitarias nacería en marzo de 1920 , siendo María de Maeztu su primera presidenta. A partir de la asunción del cargo por Clara Campoamor los objetivos se politizarán y ampliarán a temas sociales -pacifismo, acceso a la educación de clases sociales femeninas más desfavorecidas, entre otros-, coincidiendo con el compromiso político de sus miembros entre 1929 y 1936.

${ }_{89}$ Angulo, J.: «La obra de la Juventud Universitaria Femenina en España», Heraldo de Madrid, 28-9-1928, pp. 8-9. Esta reunión fue un significativo paso para los derechos femeninos en el campo universitario. Se discutió sobre la interrelación con universitarias de otros países, el intercambio de viajes y becas, y la participación femenina universitaria en particular. La amistad entre Gabriela y María debió asentarse en este momento, pues se conservan en la Residencia varias cartas de la 
EN LA FRONTERA: SEÑAS DE IDENTIDAD DE LA LABOR PEDAGÓGICA HISPANO-AMERICANISTA EN MARÍA DE MAEZTU (I924-I936)

ELVIRA M. MELIÁN

María y Gabriela ${ }^{90}$, y presumiblemente debemos al reencuentro con Blanca de los Ríos el almibarado panegírico escrito por Gabriela en 1929, donde la describe como paciente, leal y entregada, elogia su hispanoamericanismo y estudios literarios, y desafía -curioso-, a quienes la etiquetan de conservadora ${ }^{91}$.

Ya hemos comentado con anterioridad que María de Maeztu y Blanca de los Ríos son las dos únicas mujeres que figuran en la reapertura del Ateneo el in de marzo de I93I, tras su clausura en plena sublevación militar ${ }^{22}$. A esta prometedora República llega Gabriela Mistral en julio de 1933, y en ella permanecerá hasta septiembre de 1935 , cuando la prensa chilena filtre los diez folios de una carta particular emitiendo terribles críticas hacia España, siendo fulminantemente trasladada a Lisboa $^{93}$. Según su correspondencia durante esos años malvive en Madrid de sus propias colaboraciones en un clima desagradable, y en un país que aborrece. $\mathrm{Su}$ complejo carácter la mantiene alejada de los círculos de poder, cercanos a Neru$\mathrm{da}$, y busca refugio entre las mujeres del Lyceum Club, en especial Victoria Kent, a la que ha conocido a través de María de Maeztu94, y en los miembros del Comité de Cooperación Intelectual.

Aun siendo vascas de origen Maeztu y Mistral no podían ser más distintas, como describía con significativas palabras de Carlos Morla Lynch que las trató con asiduidad durante estos años:

Sus dos temperamentos, diametralmente opuestos. Gabriela es pausada, tranquila, de una serenidad austera, seca y severa, que infunde respeto al tiempo que arredramiento. Tiene la reciedumbre del granito. María es en cambio lista y avispada, de una vivacidad y aridez asombrosas. Diríase que anduviera sobre patines de ruedas. Expresa en un minuto -con una facundia prodigiosa- lo que Gabriela, con su calma reflexiva tarda un cuarto de hora en formular [...]. Posee la movilidad de mercurioss.

Esta diferencia terminará produciendo un serio enfrentamiento de causas nunca clarificadas entre 1933 y abril de $1934^{96}$. En septiembre de ese año, sin embargo, viven la «luna de miel de su reconciliación»"77, traducida en su participación

primera tras su vuelta a Argentina denominando a su anfitriona «amiga que tanto la estima y tantas cosas quiere decirle».

90 Andrenio: «La Federación de Mujeres Universitarias», La Voz, 28/9/1928, p. I.

9. Mistral, G.: «Gente española: Doña Blanca de los Ríos y Lámperez», en La tierra tiene, op. cit., p. I6I.

${ }_{92}$ Sin Firma: «Reapertura del Ateneo», op. cit.

93 Caballé, A.: «Gabriela Mistral en Madrid», Anales de la Literatura Hispanoamericana, Madrid, Ed. Complutense, 2 (1993), pp. 23I-245. Inicialmente vive en calidad de cónsul honorario sin sueldo y, desde 1935, como cónsul de segunda clase, cargo vitalicio con remuneración.

${ }_{94}$ María de Maeztu las presentaría en diciembre de 1934.

${ }_{95}$ Morla Lynch, C.: op. cit., p. 420.

96 Ibid., p. 382. Gracias a los testimonios de Morla Lynch sabemos que María, siempre conciliadora, intentará contactar con la poeta a través de amigos comunes. Durante una tensa reunión en casa de Gabriela, que continúa paseando por Retiro la intransigente chilena subraya «María yo la perdono, pero ya no la quiero. Usted ha muerto para mí y los muertos no resucitan».

${ }_{97}$ Se han barajado como causas diferencias políticas o la disputa entre Mistral y Unamuno, muy apreciado por María de Maeztu, sobre la validez o no de los indígenas para el avance de Latinoamérica, www.//hiru.com/es/literatura, pero todo son hipótesis. 
conjunta en numerosos actos que nos retrotraen al núcleo último de su hispanoamericanismo, como la creación del Hogar Americano ${ }^{98}$ y la participación en unas Jornadas hispano-americanistas en el Lyceum Club ${ }^{99}$. Pero sus actividades las distancian. Y Gabriela escribe en marzo de 1935 a Victoria Ocampo, «No he visto sino una vez y muy rápidamente a María de Maeztu. Ella y yo tenemos nuestras vidas llenas a rebosar. Y me temo que ambas, lado a lado, llenas de naderías o de cenizas de polvos duros y tristes que no dan ni fuerza ni gozo» ${ }^{100}$.

En esta misma correspondencia describe su desencuentro con España, y los motivos de su famosa carta y exilio

[...] un lío combinado de españoles patriotas (léase comerciantes) de Santiago y de unos tres colegas aficionados al cargo de Madrid facilitaron la operación de zafarme de Castilla, donde ya estaba pasando de desesperada a exasperada. La cosa no pueden entenderla nuestros hispanizantes, estilo fiesta de la raza; pero así era ${ }^{\text {IoI }}$.

Opone su amistad con María a sus encontronazos con otras amigas, y dibuja muy bien su desconcierto:

En el conflictos con los españoles, M. de Maeztu se me portó muy señorilmente, muy bellamente. La pasta es noble; las circunstancias la malean. Me temo que vuelva a sufrir mucho con la nueva situación (mucho mejor, en todo caso que la pasada). ¿Por qué no se la llevan Uds. a su Universidad por un año o por dos? Ella no lo sabe, o lo sabe, pero la tiene enferma y rota su España de contrastes brutales que la empuja de aquí a allá, haciéndola pedazos. Vea Ud., mi Victoria, por pensar en su problema, que es moral y, por rebote, físico ${ }^{102}$.

Según Ana Caballé el detonante de esta carta fue escuchar en el Lyceum Club a María Baeza relatar sus experiencias como embajadora española en Chile, posición que mantuvo su marido Ricardo Baeza desde I931, y de la que acababa de regresar ${ }^{103}$ :

Van allá estos desgraciados y Uds. Les abren clubes, hogares, salones oficiales, prensa etc. ... les dan las entrañas. Ellos vuelven contando una ciudad corrompida y grotesca, babeando aventuras de amor que son tragedias y que ellos no saben respetar, riéndose de la loca generosidad nuestra [...]. Yo no he oído a los franceses que de allá vuelven las sucias miserias que a estos desventurados charlatanes, que ni aman su sangre en la América, ni han sabido nunca que una honra personal o nacional, es una honra que se la trata con miramientos, o a lo menos sin bellaquería ${ }^{104}$.

98 Sin Firma: «Hogar americano», Luz, 16-05-1934, p. II.

99 Sin Firma: «Diplomáticas americanas en un té del Lyceum Club», El Sol, ı8-o2-1935, p. I2.

100 Mistral, G. y Ocampo, V.: Esta América nuestra. Correspondencia (1926-1956), Buenos Aires, El Cuenco de Plata, 2007.

ror Ibid., p. 56.

102 Ibid., p. 52.

103 Caballé, A.: op. cit.

104 Mistral, G. y Ocampo, V.: op. cit., p. 52. 
EN LA FRONTERA: SEÑAS DE IDENTIDAD DE LA LABOR PEDAGÓGICA HISPANO-AMERICANISTA EN MARÍA DE MAEZTU (I924-I936) ELVIRA M. MELIÁN

María de Maeztu se comportaría «muy bellamente», pero a buen seguro no pudo aprobar la acción de Gabriela siendo María Martos de Baeza su más íntima amiga ${ }^{\text {10s. }}$. De hecho, en las cartas de esos años una añorante Maeztu elogia el buen hacer de los Baeza en la embajada, habla de amistades comunes, del Lyceum Club, y se explaya sobre su estado de salud, ánimo y proyectos personales. Curiosamente en una de ellas, inédita como gran parte de las utilizadas en este estudio, se muestra de acuerdo con las que presumimos que eran críticas de María Baeza sobre las intrigas clericales en Chile, tal vez reproducidas a su vuelta y desencadenantes de la ira de Gabriela, «nada me sorprende pues algo de eso aprendí durante mi estancia en América. En España se cree que en América todo es libertad y tolerancia pero por desgracia no es así y ahí crecen, multiplicadas las rencillas de nuestra raza» ${ }^{106}$.

Entre 1935 y 1937 Gabriela Mistral permanece en Portugal, desde donde escribe a Victoria Ocampo preocupada por el destino de María al estallar la contienda civilio7, «no sería posible que Ud. ensayase Victoria el hacerle llegar desde allá este recado? Sería el que ella pudiera venirse a Portugal, conmigo, vía Galicia, en este momento [...]. No es novelería pensar que María corre peligro. La odian muchas, pero muchas, mujeres de izquierdas [...] ${ }^{108}$.

No le faltaba razón. Aunque María regresa apresuradamente desde Biarritz en julio del 1936 para retomar las riendas de la Residencia, la JAE fuerza su dimisión, dadas sus posturas cercanas a los militares ${ }^{109}$. En octubre de ese año cruza la frontera a Biarritz y desde ahí viaja a Nueva York, invitada por la Universidad de Columbia, para posteriormente establecerse en Buenos Aires, apadrinada por Victoria Ocampo. A lo largo de 1938 Gabriela y María mantienen sus contactos, pero la deriva ideológica de María abre progresivamente una brecha insalvable entre ellas. El asesinato de Ramiro de Maeztu extrema las posturas de una inconsolable hermana que, a lo largo de los años, reitera su apoyo al régimen franquista desde su exilo bonaerense, donde muere de forma repentina en $1944^{110}$. Antes, en 1945, y ya depurada sin sanción, había retornado a España en un infructuoso intento de retomar la dirección de la Residencia de Señoritas, transformada en Colegio Mayor de Santa Teresa bajo el control de la Sección Femenina de la Falange ${ }^{\text {III. }}$. Allí encontrará a Blanca de los Ríos perfectamente integrada en el Régimen ${ }^{\mathrm{II} 2}$.

Por su parte Gabriela permanecerá firme en sus posturas políticas hasta su muerte en 1957. Premonitorias palabras las que escribía a Ocampo en agosto de 1937, sobre el destino de María, y el suyo propio:

ros Melián, E. M.: 200I, op. cit.

106 CMM/ARs/Maeztu a Baeza (25/I/1932).

107 Horan, E.: «Una mixtura de calvario y arcadia. La cónsul Gabriela Mistral en Portugal, 1935-1937», Anales de la Literatura Chilena, II (2009), pp. 13-43.

${ }_{108}$ Mistral, G. y Ocampo, V.: op. cit., p. 55.

109 Ribagorda, Á.: 2006, op. cit.

no CMM/ARs/Maeztu a Baeza (4/4/1927): «Yo quiero a Ramiro por encima de todo y la conciencia de que es un caballero perfecto en un país donde tan pocos quedan él que tanto contribuyó a mi formación me reconforta el ánimo».

in Nachón Riaño, M.: «Una antigua residencia de señoritas de Madrid cumple cincuenta años», Informaciones, 24/o9/1965, p. 3.

${ }^{112}$ Villarta, A.: «Lo que desearon ser y lo que son. Doña Blanca de los Ríos», $A B C, 8 / 4 / 1945$, p. 23. 
EN LA FRONTERA: SEÑAS DE IDENTIDAD DE LA LABOR PEDAGÓGICA HISPANO-AMERICANISTA EN MARÍA DE MAEZTU (I924-I936) ELVIRA M. MELIÁN

[...] También me han dicho que María de Maeztu está con Ud. y que está fascista [...]. Salúdemela con mucho afecto [...]. Lo peor que puede pasarle es llegar a Madrid y ver el comienzo de la era de Franco. Hay que dejar que pase el estreno del fascismo en España. No durará, no puede durar y sería lamentable que ella llegase a sumarse a ese régimen de violencia y odio sombrío a la inteligencia española. Espero que ella no me dé por comunista. Me separan de ese bando todavía unas dos o tres cosas. Parecen pocas, pero cada una llega hasta el centro de la Tierra. Una es su ateísmo cien por cien, estúpido y cerrado. Pero yo no veo por donde el fascismo sea mejor calamidad, no lo veo ${ }^{113}$.

\section{Conclusión}

Es colectivamente aceptado que María de Maeztu fue un eslabón fundamental en la difusión de la ideología de la JAE en materia de educación femenina hasta 1936. Sin embargo, su contribución al nacimiento y desarrollo de un movimiento pedagógico femenino cristiano de amplia proyección hispano-americanista ha siso escasamente investigado. No es descabellado atribuir alguna responsabilidad en este silencio a su particular interpretación de esta labor como la proyección social y educativa de un ideal hispánico basado en un concepto católico-tradicionalista de la historia.

Tal vez en ninguna de las muchas iniciativas educativas acometidas por María se imbrican más profundamente la persona y la misión pedagógica como en estos años de agitación. Por eso, comprenderla exige acercarse a ella considerando la urdimbre entre ideología de la madurez, capacidad de adaptación, vocación inquebrantable, profundos afectos y contrapuestas circunstancias vitales. Este ha pretendido ser el acercamiento a uno de los fecundos desafíos de una mujer única, que acaso dejó la razón última de su destino en el párrafo profusamente subrayado por ella en rojo y acotado con la palabra iiMuy bien!!, del libro de su biblioteca personal bonaerense que ha llegado, por ventura, a mis manos:

¿Por qué no hemos de contentarnos con dejar que las compañías eléctricas y los ferrocarriles nos construyan nuestro nuevo mundo? Porque encontramos en nuestro interior sueños, hambres, aspiraciones de color, de alegría, de dignidad, de belleza, de verdad, que no incluye su programa: y somos leales a estas necesidades internas nuestras. Deseamos crear un mundo que las exprese ${ }^{114}$.

${ }^{113}$ Mistral, G. y Ocampo, V.: op. cit., p. 60.

${ }^{114}$ Frank, W.: Primer mensaje a la América hispana, Madrid, Espasa Calpe, 1932, p. 287. 